\title{
The application of animal-assisted therapy in the South African context: A case study
}

\section{Carien Lubbe and Suné Scholtz}

\begin{abstract}
The purpose of this article is to explore the use, value, and applicability of animal-assisted therapy in psychology. The case study method was applied to a therapeutic case, using the interpretivist paradigm. Data were analyzed by means of document analysis. The findings are discussed according to five themes derived from the study, namely, "facilitating relationship building," "enabling communication by working indirectly," "experiencing physical affection through the therapy dog," "socialization skills," and "enhanced self-esteem." We report that the deliberate inclusion of an animal in therapy facilitates relationship building and therefore aids the therapeutic alliance, as well as enhances an individual's socialization skills and self-esteem.
\end{abstract}

\section{Keywords}

Animal-assisted therapy, case study, child, document analysis, psychology

The use of animal-assisted therapy (AAT) is increasing globally (Chandler, 2005). The literature on AAT suggests that animals benefit humans, both directly and indirectly. Although research has increased over the past couple of decades, the existing literature is quite limited. Up to 2004, there were approximately 40 studies examining the efficacy of animal-assisted interventions (Nimer \& Lundahl, 2007).

AAT can be defined as a goal-directed intervention in which an animal is a part of the treatment process delivered by a health/human service professional (Anderson, 2004; Hettema, 2002; Kruger \& Serpell, 2006; Odendaal, 2002; Tedeschi, Fitchett, \& Molidor, 2006). Nimer and Lundahl (2007) write that AAT is "the deliberate inclusion of an animal in a treatment plan" where "the introduction of the animal is designed to accomplish predefined outcomes believed to be difficult to achieve

University of Pretoria, South Africa

\section{Corresponding author:}

Carien Lubbe, Department of Educational Psychology, Faculty of Education, University of Pretoria, Groenkloof Campus, All Purpose Building, CII4, Pretoria 000I, South Africa.

Email: carien.lubbe@up.ac.za 
otherwise or outcomes best addressed through exposure to an animal" (p. 264). This indicates that the use of an animal in AAT is intentional and that the animal may help the therapist to achieve outcomes in challenging therapy cases more easily.

Numerous authors have argued for the use of animals in therapy. For example, Nimer and Lundahl (2007) found that animals seem to have a natural affinity for creating a relationship (bond) with people, thus leading to the possibility of AAT being beneficial. Chandler (2005) states that this natural tendency of animals fosters quick rapport and empathy between children and animals. According to Urichuk and Anderson (2003), all humans have basic psychological needs (such as being loved, respected, useful, needed, accepted, and trusted) that an animal can meet by fulfilling important roles. These roles include companion, friend, servant, dependant, admirer, confidante, scapegoat, mirror, trustee, and defender (Urichuk \& Anderson, 2003).

A very important bond may be formed between a therapy animal and a person in therapy. This bond may help to gain the client's trust, since the client may trust the therapy animal well before trusting the therapist. Chandler (2005) mentions that although the relationship between a therapy animal and a client is not meant to be a substitute for the relationship between the human therapist and the client, the therapy animal may serve as a transitional object onto which clients can project their feelings and subjective experiences. Furthermore, Fine (2006) indicates that animals promote rapport in a therapeutic relationship, which is important in establishing trust in a therapeutic relationship.

\section{Animal-assisted therapy (AAT)}

Odendaal (as cited in Hettema, 2002) maintains that there are two motivations for human-animal interaction. The first is to elicit emotional advantages and the second is the utility value for humankind. The latter refers to sport and recreation, working, and as a form of security. In this article, we focus on the former, by exploring how AAT can be used as a therapeutic tool in psychology.

According to definitions developed by the Delta Society (2009), therapy with an animal can be divided into two broad types, animal-assisted activities (AAA) and AAT. AAA entails less formal human-animal interaction and is mostly used for social visits with a therapy animal (Chandler, 2005). As previously mentioned, AAT refers to a goal-directed intervention, where the animal meets specified criteria (Anderson, 2004; Hettema, 2002; Kruger \& Serpell, 2006; Odendaal, 2002; Tedeschi et al., 2006). The Delta Society (2009) further mentions that AAT is scheduled at regular intervals and that notes on the client's progress are made after each session.

Hettema (2002) notes that this specific intervention takes place within environments such as prisons, nursing homes, homes for the disabled, and in childcare settings. Jalango, Astorino, and Bomboy (2004) are of the opinion that therapy dogs, for example, provide a unique form of support for children's learning, physical health, and emotional well-being, which may not have been possible through interaction and intervention with the therapist alone. Friesen (2010) affirms that by incorporating a dog into the therapy setting, interaction between a child and adult may become possible where human attempts alone have been proven to be inadequate, pointing out that AAT is a unique form of support.

The possibility of applying AAT within the helping professions is therefore explored in this article, as it is important to consider new techniques that can be used and tested for efficacy in research, and to investigate the value of any particular technique or approach. AAT has not been widely researched, especially in the South African context, since it is a relatively new field. Furthermore, there is a lacuna in research on AAT with children. 


\section{Background to the research study}

The second author (S.S.) first observed AAT during visits to a nearby hospital, and found that the clients who had been treated using AAT appeared to be more positive and lively, and it seemed that they temporarily forgot about their physical pain. This resonated with Fine's (2006) findings that interaction with animals lightens the mood and increases smiling and laughter in clients. The adults and children in the hospital appeared to have enjoyed the company of the therapy dogs, and demonstrated this enjoyment by stroking them or feeding them biscuits. As part of the preparation for using the dog (Morkie) in AAT, as well as to learn to be aware of the dog's needs, S.S. worked through a program with an animal behaviorist. AAT has several specifications to which we adhered. The dog should have the right temperament, and be friendly, affectionate, and sociable with all ages and ethnicities, as well as any gender (Chandler, 2005). The dog should also be able to tolerate high levels of noise and activity and be relatively calm, obedient, and easy to control. Chandler (2005) also refers to the fact that the dog should be comfortable traveling in a car and visiting unfamiliar places, and also be able to tolerate stress. By working with an animal behaviorist, we were able to ensure that these criteria were met.

In this study, we hold the assumption that children would feel more at ease when in the company of a dog, and therefore find it easier to project their feelings onto the dog and tell the dog "secrets" they would not normally tell a therapist. Throughout the therapeutic process, the presence of Morkie seemed to be a significant factor in making progress with the therapy. This prompted the research study to explore the possible value of AAT.

\section{Method}

In this study, we explored the use, value, and applicability of AAT with a child in a therapeutic setting by conducting a document analysis of the case file of a child whom we had worked with earlier. In the following sections, we provide a synoptic overview of the case file, as well as an outline of the nature of the inquiry.

\section{Background to the case study}

The client, Brandon (a pseudonym), was a 14-year-old boy in Grade 6, placed in a place of safety in an urban setting. Brandon was admitted to a place of safety by his mother, and his father was incarcerated. He had refused to attend school, and it was not known what Brandon did when not at school. Furthermore, his face was scarred as a result of a benzene bomb that exploded in his face when he was younger. He had already attended therapy before attending therapy with us, but the previous therapist mentioned that she could not work with him because he did not show her any respect and was also unwilling to interact with her.

The following therapeutic goals were established as part of the treatment plan, namely, selfconfidence and self-worth, self-acceptance, trust in others, success experiences, school attendance and participation, social skills, the mother-child relationship, and family functioning. During the first few sessions, it was challenging to establish an effective therapeutic relationship with Brandon, as he did not talk or answer questions or make eye contact. However, Brandon reacted positively toward Morkie when she happened to have joined in the therapy.

\section{Research design}

A qualitative methodological approach, which was epistemologically anchored in interpretivism, was used in this study. We employed an intrinsic case study design, focusing on the aim of gaining 
an enhanced understanding of the individual case (De Vos, Strydom, Fouché, \& Delport, 2005). This allowed us to gain a deeper understanding of the case under investigation. The individual case is therapeutic intervention with a 14-year-old boy whom S.S. engaged with as part of her master's training in Educational Psychology using AAT in the South African context.

\section{Data sources and analysis}

The data involved the various sources as captured in the therapeutic case file, namely, a book made by the participant during therapy, which contained letters, drawings, paintings, and photographs. In addition, we returned to the participant a year and a half after the termination of therapy, in order to obtain a sense of what the sustainability of the AAT process was, as well as the meanings he could possibly assign to the therapy process in hindsight. In this data gathering session, we made use of an informal interview, narrative expression (letters), and creative expression (drawings/ paintings) to demonstrate his experiences of the therapy process.

We conducted the data analysis by means of document analysis. Denzin and Lincoln (2005) clarify that document analysis refers to the procedures involved in analyzing and interpreting data obtained from the examination of documents relevant to the study. Similarly, Punch (2005) describes document analysis as the systematic examination of documents in order to identify needs and challenges and to describe an activity. The focus of the analysis should be a critical examination, rather than a mere description, of the documents. Document analysis works best when the purpose is to gain insight into an activity or approach. He further describes the use of documentary data for social research as a "rich" process. It is important to take the context in which documents have been written into consideration, as they may be divested of their real meaning if viewed in isolation from their social context (Punch, 2005). We analyzed and interpreted the letters, drawings, paintings, and photographs that were in the therapeutic case file. This process allowed us to identify certain common themes that were present in all of the data collected.

\section{Ethical considerations}

We obtained informed consent in order to ask whether we could use this therapeutic case for research purposes. As the child was underage and evidently a child with special educational needs, we needed to apply caution and continuously reflect on whether we were acting in the best interests of the child. We presented the participant and his mother with letters of consent and assent to sign in which the research process was described. We explained the purpose of the research and the interview that would be conducted, as well as the follow-up conversation with him. In addition, an informed consent form signed by the university's Animal Use and Care Committee also had to be completed regarding the use of the dog as a therapeutic medium. On this form, the process involved was explained, as well as the possible risks for the animal in the therapeutic process.

The aforementioned consent form made provision for permission to take and display photographs of the participant. Although the participant and his mother did not mind the photographs being used, we decided to minimize the possibility of identifying the participant by superimposing a bar over his eyes in the photographs and covering his name in the letters and drawings. This research study did not entail exposing the participant to physical risks or harmful activities. On a psychological level, we were mindful of the fact that this is a vulnerable child, and therefore, we attempted not to cause any harm and to establish a caring relationship without compromising the research findings. 
The rights of the animal were protected by preventing hunger, thirst, malnutrition, fear, distress, physical or thermal discomfort, pain, injury, and disease, and also by allowing the animal to exhibit normal patterns of behavior. This was done through personal knowledge obtained through training the dog, as well as observing the animal during the therapy sessions.

\section{Findings and discussion}

The themes from the document analysis were interrogated by confronting the themes with analytical questions of meaning and significance, which Mason (2002) terms "holistic analysis." We asked ourselves, "What is the significance of using AAT as a therapeutic approach, for this case specifically but perhaps more importantly, for therapy in a broader sense?" and "What benefits and values can be found in applying AAT?" Five themes were identified in the analysis, namely, "facilitating relationship building," "enabling communication by working indirectly," "experiencing physical affection through the therapy dog," socialization skills," and "enhanced self-esteem."

\section{Theme I: facilitating relationship building}

When the therapist met Brandon for the first time in therapy, he seemed to be a very shy boy who did not talk much, merely responding with simple yes or no answers. He sat in front of the therapist, looking down at his lap, with his hands folded. Brandon always wore a sweatshirt top with a hood, which was pulled over his head, covering half his face. During the session, he avoided eye contact with the therapist and did not answer any questions or initiate any conversation, indicating that he may have felt uncomfortable with her and did not trust her at that time, which is typical and to be expected during therapy in the initial phases, but was experienced as extremely frustrating by the therapist. This behavior continued for the duration of the first three sessions. During the fourth session, the therapist took her therapy dog, Morkie, along. Although, on that first occasion, Brandon did not interact with Morkie, he smiled at her as she stood up against his legs, looking up at him.

Friesen (2010) and Chandler (2005) affirm that including a dog in therapy may facilitate the relationship between the child and the therapist, indicating that by using a dog, the therapist may be assisted in establishing a relationship with the client. Several researchers have found that having an animal present in the therapy setting makes the therapist less threatening and more approachable, while also decreasing the client's initial reservations about entering therapy. This results in the client being more willing to reveal himself or herself (Fine, 2006; Urichuk \& Anderson, 2003).

After a few weeks of therapy with Morkie, Brandon seemed to be more open towards the therapist. He looked her in the eye when he greeted her, smiled at her, spoke to her, and also answered questions directed indirectly from Morkie (see Figure 1). Schaifer (1999) states that the threatening feeling of being under examination is forgotten when an animal is included in therapy. This indicates that the therapy dog decreases some tension, creating an inviting atmosphere, while also shifting the client's attention from being worried to feeling calmer and relaxed. Fine (2006) maintains that the presence of a dog in therapy reduced the initial tension and created an atmosphere of warmth.

During previous sessions, Brandon used to turn his back on the therapist when writing a letter to Morkie. During Session 11 (Figure 2), Brandon came to sit opposite her to write his letter to Morkie. This gesture may indicate that Brandon had developed trust, was comfortable with the therapist, and may have felt more able to communicate with her. In common with Urichuk and Anderson's (2003) finding that animals promote therapeutic disclosures and enhance therapeutic progress, this may 


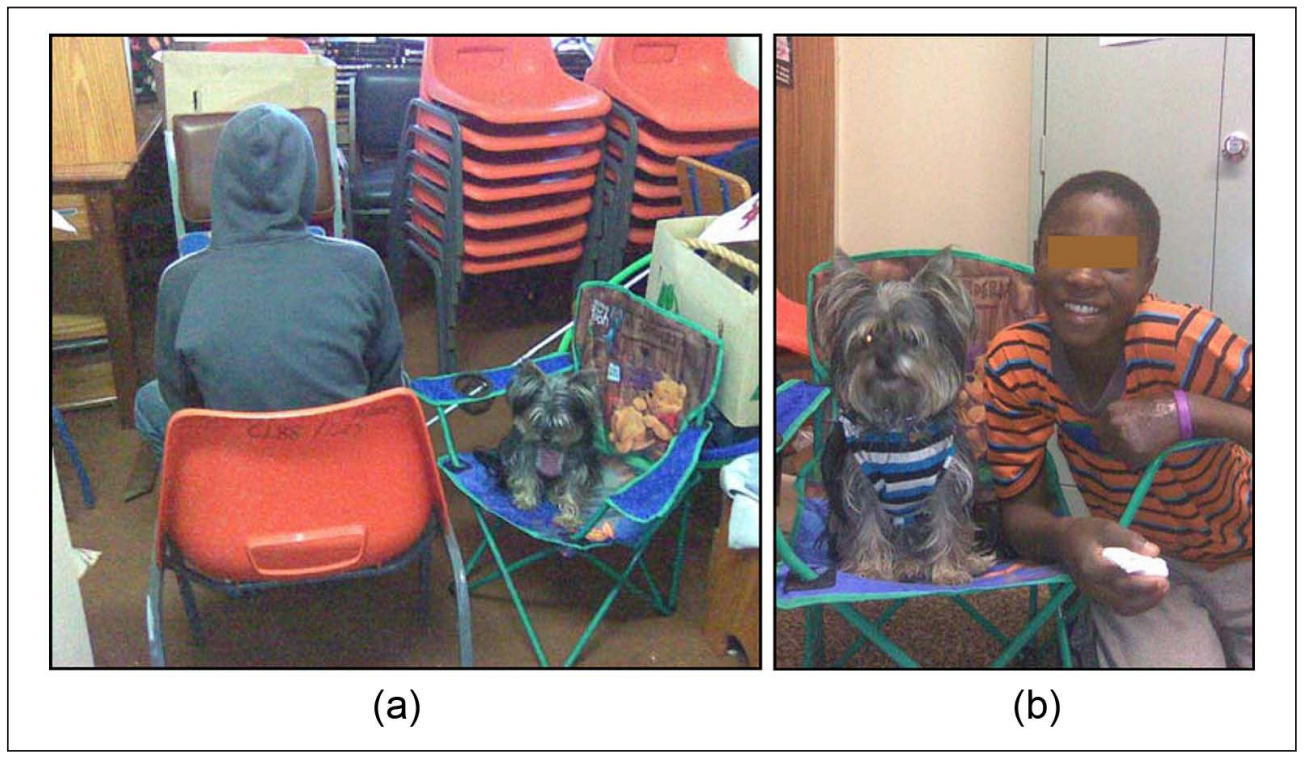

Figure I. Brandon seems to be more open toward the therapist in (b) Session 6 compared to (a) Session 5.

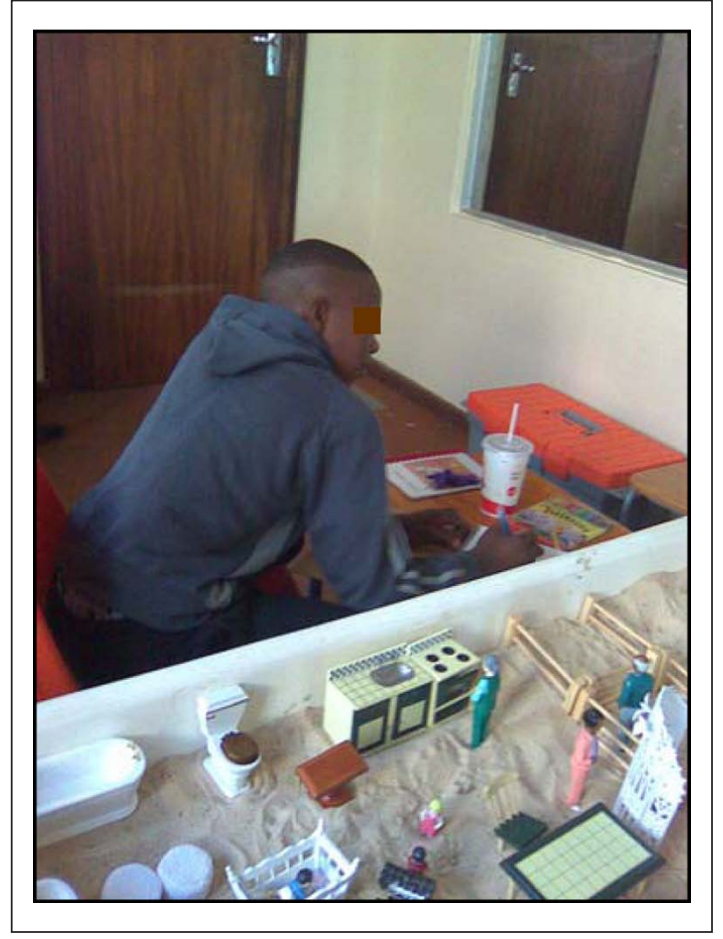

Figure 2. Brandon's posture change in Session II. 
indicate that Morkie's presence in the therapeutic setting not only enhanced therapeutic progress but also made Brandon feel more relaxed about the presence of the therapist.

Using Morkie in therapy gave the therapist an opportunity to develop a deeper relationship with Brandon. We believe Morkie not only served as a motivational tool for therapy but that she also improved the therapeutic relationship in several ways. The therapist had the opportunity to interact with Brandon through the medium of the dog, which he may have found less threatening, also allowing the therapist herself to give him positive feedback and affirmation. Using Morkie, the therapist gave Brandon some responsibility by getting him to help take care of her, indicating that she trusted him. This responsibility included filling Morkie's bowl with water, putting a leash on her, and placing her in her travel cage. These responsibilities gave Brandon a sense of enhanced self-efficacy, independence, and control.

Fine (2006) maintains that an animal's presence allows the client a sense of comfort, which in turn promotes rapport between therapist and client. Tedeschi et al. (2006) support the findings in that they describe that when the client creates an initial trust connection with an animal, it may be transferred to the professional at a later stage. As a result of this, one may conclude that an animal ( dog) may assist a therapist in creating a relationship with a child (client). Fine (2006) states that a gentle animal helps the client to view the therapist more favorably, as the child may notice that the dog, which he or she trusts, has a good relationship with the therapist, which may result in the child trusting the therapist at a later stage as well. We believe that this, too, may be one of the reasons Morkie facilitated the establishment of a good relationship between Brandon and the therapist.

Nimer and Lundahl (2007) describe a "good" therapy animal as one that would seek attention and affection from the child. It was found that animals intentionally show certain behaviors (Chandler, 2005) such as physical contact with a child, but more importantly, they never contradict the attributes projected onto them in words (Katcher, 2000, as cited in Chandler, 2005). This may result in the child experiencing the animal as "unconditionally accepting and non-judgmental" (Chandler, 2005, p. 6). Morkie frequently sought attention from Brandon in that she would walk up to Brandon, lean against him, and look up at him. Brandon enjoyed this very much as he smiled and relaxed in his posture. As mentioned previously, she also climbed onto the table while Brandon was busy working, which may have made him feel important, as he received special attention through this physical contact.

Soloman (as cited in Reichert, 1998) maintains that the child receives a certain value from a dog that offers love and reassurance without criticizing the child. During a specific session, Brandon revealed that he was sad as a result of something his mother had done. Morkie appeared to sense the sadness and climbed onto his lap. This gave the therapist the opportunity to tell Brandon that Morkie cared, thus causing him to feel special and important. Tedeschi et al. (2006) state that "animals can often reach people when human relationships may be difficult" (p. 62).

Theme 1 shows that the presence of an animal may be useful, especially when the child does not feel safe or mistrusts the therapist. The animal (dog) may form a relationship with the child, as it would not pose a threat to him or her. Fine (2006) found that children were more "relaxed and seemed more cooperative" (p. 173) at times in the presence of a dog, thus being a particularly helpful strategy (Schaifer, 1999). Brandon seemed to become more and more cooperative in that he started to participate in the sessions by answering questions, talking, and even allowing the therapist to take photographs of him and Morkie.

\section{Theme 2: enabling communication by working indirectly}

Urichuk and Anderson (2003) indicate that an animal as co-therapist may facilitate communication. During therapy with Brandon, the therapist used Morkie to communicate with him in several ways. 
First, she pretended that Morkie could speak or that she could hear her if she listened closely enough. The therapist simply acted as the agent, "relaying the message to the dog or the child" (Schaifer, 1999, p. 420), by, for example, carefully stating that "Morkie says . . " This enabled Brandon to communicate with the therapist using Morkie as a medium and, thus, not feeling threatened or exposed. This gave Brandon the opportunity to communicate more freely, although it still provided him with a protective barrier, as it was Morkie "speaking" or "telling" the therapist something, not him. He frequently made choices or told the therapist things from his personal life by using Morkie as a medium. In asking questions through Morkie, Brandon started to answer them, whereas previously, he only used yes or no answers. This may have been because it was the dog "asking" the questions or it may also have been that Brandon had started to trust Morkie and to feel more at ease, feeling that she accepted him unconditionally, although we cannot prove or measure whether he felt that Morkie accepted him unconditionally. Second, the therapist made use of letters between Brandon and Morkie (see Figure 3). Brandon reacted very positively to this in that he wrote back to Morkie. He had found a way to communicate, and this resulted in one of the main ways in which we communicated. Brandon expressed his feelings, fears, likes, and dislikes in these letters to Morkie. "Studies have shown that children regularly confide in their pets when they have a problem, and play with their pet when feeling stressed" (Covert, Whiren, Keith, \& Nelson, 1985, as cited in Urichuk \& Anderson, 2003, p. 52). Although the above research found that children confide in their pets, one may assume that they trusted the animal, and also felt safe and relaxed in its presence.

As we returned as researchers to meet with Brandon, he asked whether he could write a letter to Morkie, indicating the attraction this manner of communication held for him. Both his mother and Brandon himself mentioned that he felt he could talk to Morkie, as well as to the therapist, showing that Morkie had most likely assisted Brandon in communicating.

Urichuk and Anderson (2003) found that "personal development, such as speech and communication skills" (p. 79) can be improved by working with an animal. The therapist asked Brandon to respond with "good dog" every time Morkie did something he was pleased with, in order to get him to talk and to start interacting. This modeled the behavior one uses to interact with another person in terms of giving them positive feedback. Furthermore, this may also have provided Brandon with the opportunity to socialize with someone using verbal communication, as he had not, at that time, communicated much with the therapist. As time passed, Brandon started to interact with the therapist more and more, making eye contact with her, smiling at her, and speaking to her. However, he still needed Morkie in order to answer questions indirectly, although all that changed in the following sessions. Brandon had started to answer the therapist's questions directly, without hesitation, indicating that Brandon started to feel competent enough to interact in a social manner. We do feel that Morkie's presence helped Brandon reach this point as she never criticized him, always listened to him, and was a safe medium through which he could express himself without feeling exposed. We also feel that the therapist would not have been able to achieve this in such a positive manner without the assistance of Morkie in therapy.

Urichuk and Anderson (2003) found that individuals may benefit from hearing stories of the animal's experiences that are similar to their own issues. During therapy, the therapist made use of storytelling and projection in order to work more indirectly with Brandon. She ensured that the story was about Morkie but that it was based on similar events in Brandon's life. The therapist made use of projection in order to find solutions for Morkie, transferring the pressure from Brandon, causing him to be more actively involved. Brandon identified strongly with this and frequently gave Morkie advice, also using some of the advice for himself. As Reichert (1998) states, "By promoting projection and identification of the child's feelings, storytelling with the animal can be beneficial to the therapeutic process" (p. 184). As therapy progressed, Brandon started to grow 


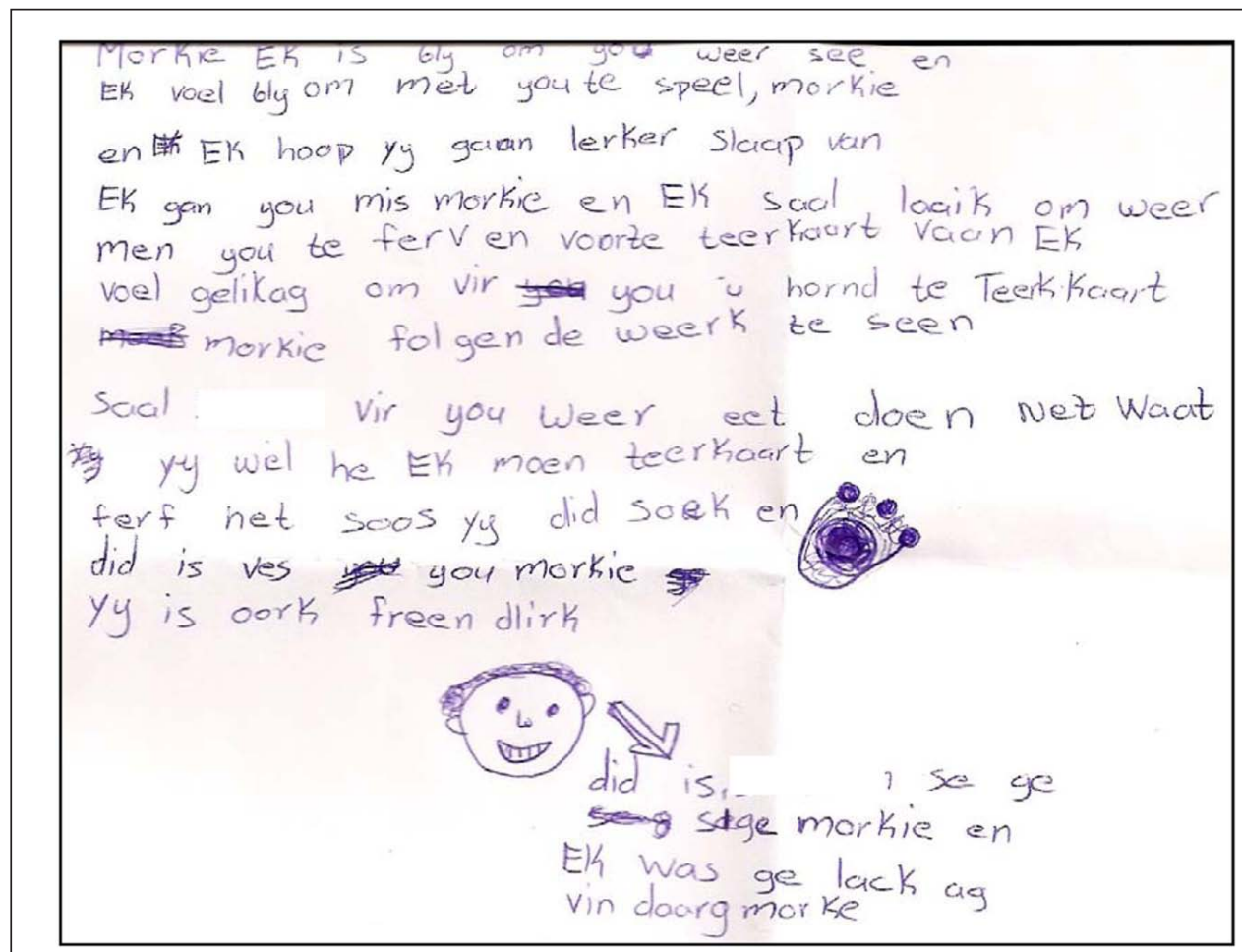

The following is a translation from the Afrikaans:

Morkie I am happy to see you again and I feel happy to play with you, Morkie and I hope you will sleep nicely.

I am going to miss you Morkie and I would like to paint with you again and to draw for you.

I felt happy to draw a dog for you Morkie. Next week see again.

Brandon will do just what you want me to draw and paint, just as you want it and that is for you Morkie.

You are also friendly.

This is Brandon's face Morkie and I was happy today Morkie.

Figure 3. An example of a letter Brandon wrote to Morkie.

more independent of Morkie. He did not need to use Morkie as a projection medium any more nor did he hesitate to talk directly to the therapist. This was evidence to us that, by working indirectly, using projection and storytelling in conjunction with an animal, the client dealt with issues in a safe, indirect manner, also leading to him becoming more independent.

The therapist also used Morkie to prepare Brandon for situations that he might need to deal with in the future, asking him how we could help or support Morkie. This technique was successful because Brandon suggested the use of a coping mechanism we had developed for Morkie in a previous session, indicating that he too had acquired coping skills through the therapy. This may have been an indication of how Brandon would have reacted if he was in the same situation as he may have associated himself with Morkie, indicating that by working indirectly with the dog, he may have acquired the skills needed to cope with his situation. Reichert (1998) states that "because a 
child's ability to identify with characters and themes is so powerful, the child can easily relate to a story and make unconscious connections to heroes and conflicts" (p. 187). Using a story may thus help a child to identify with a character, leading to the resolution of his or her problems.

\section{Theme 3: experiencing physical affection through the therapy dog}

During most of the therapy sessions, Brandon would start off by stroking Morkie, giving her some love and attention. This was very important to Brandon, and one could see that he cherished the physical contact with Morkie. Morkie frequently went up to Brandon and looked at him, indicating that she was interested in him, always making him smile. Using Morkie also provided the therapist with the opportunity to use her in activities that Brandon was involved in, such as painting or drawing. She frequently climbed onto the table, sitting next to Brandon as he was painting (see Figure 4), giving him the impression that they were doing the activity together and also that she was interested in him and what he was doing.

Brandon enjoyed this physical interaction as he frequently touched her (see Figure 5), allowed her onto his lap, and pulled Morkie's chair closer to his, indicating the possibility that he longed for physical closeness between Morkie and himself.

Allan (2008) cautions that physical contact between a client and a therapist is a high-risk activity. This may be why animals are in a unique position to display emotions and behaviors that may not be deemed appropriate for mental health therapists (Urichuk \& Anderson, 2003). Owing to the society we live in and the need to adhere to certain ethical standards, mental health professionals should avoid physical contact with their clients. Urichuk and Anderson (2003) claim that contact with animals is a safe way in which the individual is able to experience the physical and emotional benefits of touch.

\section{Theme 4: socialization skills}

In the beginning, the therapist told Brandon that he should hold out his palm toward Morkie in order for him to indicate that he was friendly and wanted to be friends, and that he would not hurt her. This

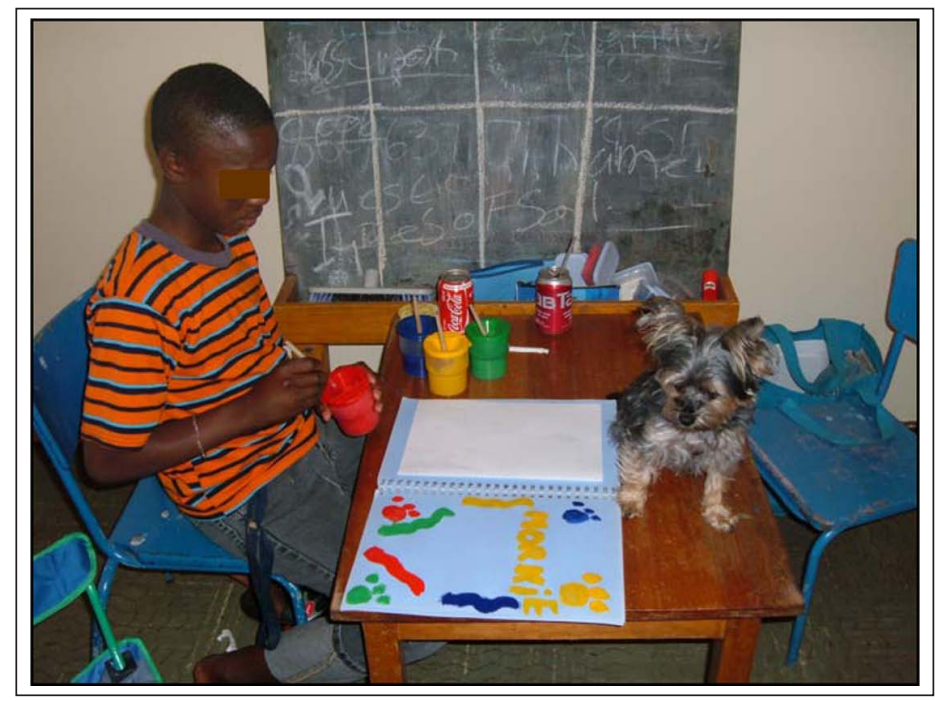

Figure 4. Morkie's interaction with Brandon during therapy. 


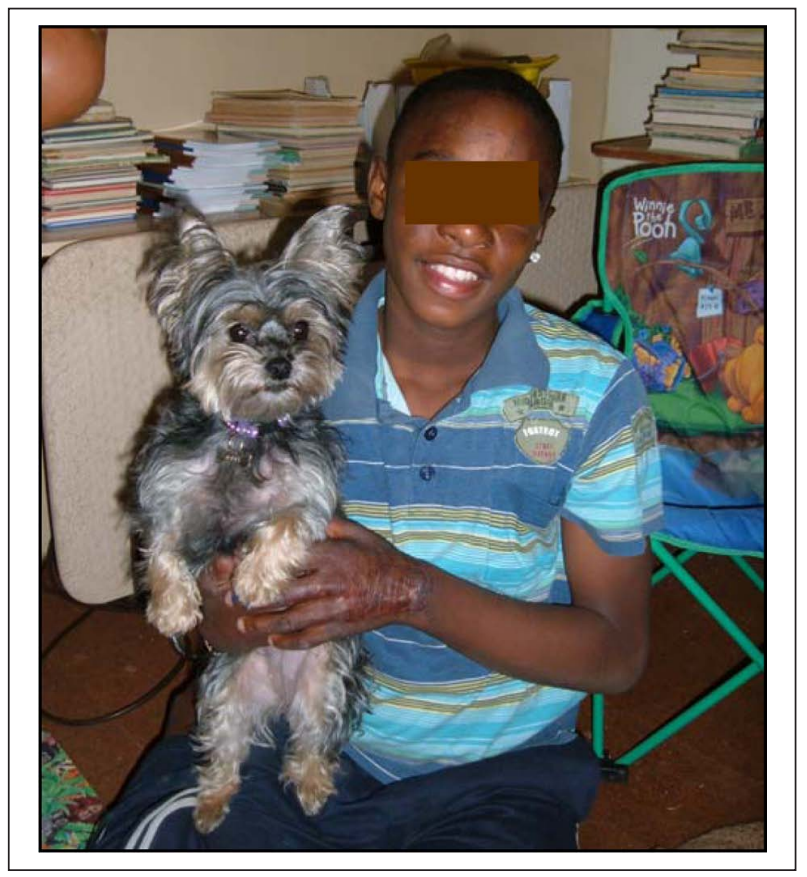

Figure 5. Brandon's physical interaction with Morkie.

may have been a basic gesture toward a dog; however, it had a much deeper meaning. Indirectly, Brandon had the opportunity to reach out to someone as a social gesture, thus practicing what it would be like going up to someone and greeting them. The possibility exists that there might be some transference between an animal object and the underlying need to "be" with someone.

Urichuk and Anderson (2003) maintain that young children and some adults see animals as peers. This may promote the interaction between people and animals, indicating the importance of AAT. During the following session, Brandon held out his palm to Morkie immediately. He not only received immediate positive feedback from Morkie in that she came toward him but also verbal affirmation from the therapist, affirming that when you reach out to someone in a calm way, you will be treated in the same way in return.

Brandon started to direct questions toward Morkie through his letters to her, which may have indicated social skills in that Brandon was initiating a conversation with Morkie. He increased his awareness of social skills in that he noticed Morkie was friendly toward him and that he would need to be friendly in return for their friendship to develop. Brandon also started to use words to describe his feelings, indicating that communicating with Morkie had increased his ability to express his feelings, as well as to establish social networks in the Place of Safety. As mentioned before, Brandon was quite hesitant about interacting or socializing with others. However, after some time in therapy, he started to greet one of the other therapists spontaneously, whereas previously she had had to greet him first. This indicates that therapy with Morkie had enhanced Brandon's socialization skills. Urichuk and Anderson (2003) found that when people are taught to be kind to animals and to treat them with respect, they also learn to be kind and to respect other people. Many researchers have found that using an animal in therapy enhances social support and social interactions (Brodie \& Biley, 1999; Friedmann \& Tsai, 2006; Kaminski, Pellino, \& Wish, 
2002; Shiloh, Sorek, \& Terkel, 2003; Tedeschi et al., 2006). McIntosh (2002, as cited in Urichuk \& Anderson, 2003) asserts that children may often turn to animals for social support, on account of the animal's unconditional acceptance of the child and being nonjudgmental toward him or her.

\section{Theme 5: enhanced self-esteem}

Interacting with Morkie may have made Brandon feel more positive and accepted and may have raised his self-esteem in that someone wanted to be with him. Additionally, using Morkie in therapy allowed the therapist to praise Brandon for his actions and interactions with her, which might also have increased his self-esteem. Furthermore, Brandon was asked to give Morkie certain commands in order for her to perform obedience tricks. This required Brandon to speak louder than he usually did, also using a little more authority. This, in turn, allowed Brandon to experience some control as well as the possibility of increasing his self-esteem.

Using a dog in therapy gave the therapist the opportunity to provide Brandon with more responsibility during the sessions. As mentioned before, he had to pour water into Morkie's bowl, as well as choose an activity to start with during therapy. Another way in which AAT provided Brandon with more control and power was walking Morkie on a lead to the nearest park, which may have made him feel proud of being able to walk her. This also gave him some self-confidence, as Morkie listened to him and responded to his commands. These actions and responsibilities may have empowered Brandon to a certain extent, also allowing the therapist an opportunity to show that she trusted Brandon to carry out these tasks, ultimately leading to an increase in his self-esteem and self-confidence. As therapy with Brandon and Morkie progressed, we noticed that he started to be more creative in his work, indicating that he may have felt more confident to try out new things.

Research has found that children who have regular contact with animals have higher levels of selfesteem (Urichuk \& Anderson, 2003), as they provide unconditional positive regard, do not discriminate, are nonjudgmental, and do not play psychological games with people, which makes them ideal for increasing a person's self-esteem. They also mention that the animal "loves you for who you are inside" (Urichuk \& Anderson, 2003, p. 80), which may convey unconditional acceptance from the animal to the child. Similarly, Reichert (1998) found that because the animal is nonjudgmental, the child's self-esteem is enhanced and expression of feelings may be promoted. Urichuk and Anderson (2003) found that clients who lack a sense of control and self-esteem in their lives may benefit from AAT. Tedeschi et al. (2006) found that by working with an animal, the child is allowed the opportunity to "gain more control and feel unconditionally accepted by a living being" (p. 69).

\section{Limitations and recommendations}

A major drawback of AAT is that it entails the use of a properly trained animal, as well as familiarity with AAT, which may imply that the applicability and transferability to a wide scope of practitioners might be limited. Furthermore, the possibility (and probability) exists that various other factors might have impacted on the findings that we obtained. The use of AAT may therefore not be seen as the sole contributor to the participant's emotional healing. As such, the positive therapeutic relationship, ongoing therapeutic sessions, the establishment of other meaningful relationships, regular contact with the participant, and his natural emotional and social development, might also have been of therapeutic value and could have impacted on the progress during therapy.

As mentioned previously, it appears that limited research has been undertaken in the field of AAT as a therapeutic tool. Therefore, we recommend that further studies be conducted in this field 
of research, with the purpose of broadening and improving the current knowledge base. The following areas of interest may be explored in future research, namely, research that explores the application potential of AAT with a larger sample of socioeconomically vulnerable children, and the possibility of social workers, play therapists, and psychologists being trained in and applying AAT in therapy in places of safety, schools, and private practice.

\section{Concluding comments}

Reflecting on our findings, it appears that AAT could indeed be used successfully as a therapeutic tool in psychology. The use of AAT in the therapeutic setting provided Brandon with a way to communicate, express himself, and acquire the needed coping mechanisms.

From the findings, however, it can be concluded that using an animal (dog) in the therapeutic process appears to promote the formation of a personal and caring relationship with the child. This is especially valuable with a child who was not keen on establishing a relationship with the therapist at first, and the dog provided a safe medium through which support could take place. This supports the overall goal of any therapeutic process to enable an individual, in this case a child, to deal with painful emotional issues; to achieve some level of congruence with regard to thoughts, emotions, and behaviors; to feel good about himself or herself; to change behaviors that have negative consequences; and to function comfortably and adaptively within the external environment (Geldard \& Geldard, 2002). Overall, it seems as if AAT can be purposefully applied to support the achievement of therapeutic goals.

\section{Declaration of conflicting interest}

The authors declare that they do not have any conflict of interest.

\section{Funding}

This research received no specific grant from any funding agency in the public, commercial, or not-for-profit sectors.

\section{References}

Allan, A. (2008). Law and ethics in psychology: An international perspective. Somerset West, South Africa: Inter-Ed.

Anderson, D. C. (2004). The human-companion animal bond. The Reference Librarian, 41, 7-23.

Brodie, S. J., \& Biley, F. C. (1999). An exploration of the potential benefits of pet-facilitated therapy. Journal of Clinical Nursing, 8, 329-337.

Chandler, C. K. (2005). Animal-assisted therapy in counseling. New York, NY: Routledge/Falmer.

Delta Society. (2009). What are animal-assisted activities/therapy? Retrieved from www.DeltaSociety.org/ AnimalAssistedActivitiesTherapy

Denzin N. K., \& Lincoln Y. S., (Eds.). (2005). The SAGE handbook of qualitative research (3rd ed.). Los Angeles, CA: SAGE.

De Vos, A. S., Strydom, H., Fouché, C. B., \& Delport, C. S. L. (2005). Research at grass roots: For the social sciences and human service profession (3rd ed.). Pretoria, South Africa: Van Schaik.

Fine, A. H. (2006). Incorporating animal-assisted therapy into psychotherapy: Guidelines and suggestions for therapists. In A. H. Fine (Ed.), The handbook of animal assisted therapy (pp. 167-206). San Diego, CA: Academic Press.

Friedmann, E., \& Tsai, C.-C. (2006). The animal-human bond: Health and wellness. In A. H. Fine (Ed.), Animal-assisted therapy: Theoretical foundations and practice guidelines (2nd ed., pp. 95-117). San Diego, CA: Academic Press. 
Friesen, L. (2010). Exploring animal-assisted programs with children in school and therapeutic contexts. Early Childhood Education Journal, 37, 261-267.

Geldard, K., \& Geldard, D. (2002). Counselling children: A practical introduction (2nd ed.). Thousand Oaks, CA: SAGE.

Hettema, E. (2002). Animal-assisted therapy: What is it? (Unpublished master's thesis). University of Stellenbosch, Stellenbosch, South Africa.

Jalango, R. M., Astorino, T., \& Bomboy, N. (2004). Canine visitors: The influence of therapy dogs on young children's learning and well-being in classrooms and hospitals. Early Childhood Education, 32, 9-16.

Kaminski, M., Pellino, T., \& Wish, J. (2002). Play and pets: The physical and emotional impact of child-life and pet therapy on hospitalized children. Children's Health Care, 31, 321-335.

Kruger, K. A., \& Serpell, J. A. (2006). Animal-assisted interventions in mental health: Definitions and theoretical foundations. In A. H. Fine (Ed.), The handbook of animal assisted therapy (pp. 21-38). San Diego, CA: Academic Press.

Mason, J. (2002). Qualitative researching (2nd ed.). London, England: SAGE.

Nimer, J., \& Lundahl, B. (2007). Animal-assisted therapy: A meta-analysis. Anthrozoös, 20, 225-238.

Odendaal, J. (2002). Pets and our mental health: The why, the what and the how. New York, NY: Vantage Press.

Punch, K. F. (2005). Introduction to social research: Quantitative and qualitative approaches (2nd ed.). London, England: SAGE.

Reichert, E. (1998). Individual counselling for sexually abused children: A role for animals and storytelling. Child \& Adolescent Social Work Journal, 15, 177-185.

Schaifer, C. (Ed.). (1999). Innovative psychotherapy techniques in child and adolescent therapy (2nd ed.). New York, NY: John Wiley.

Shiloh, S., Sorek, G., \& Terkel, J. (2003). Reduction of state-anxiety by petting animals in a controlled laboratory experiment. Anxiety, Stress, \& Coping, 16, 387-395.

Tedeschi, P., Fitchett, J., \& Molidor, C. E. (2006). The incorporation of animal-assisted interventions in social work education. Journal of Family Social Work, 9, 59-77.

Urichuk, L. J., \& Anderson, D. (2003). Improving mental health through animal-assisted therapy. Edmonton, Alberta, Canada: The Chimo Project. 